\title{
Collusion-Resistant Domain-Specific Pseudonymous Signatures ${ }^{\star \star \star}$
}

\author{
Julien Bringer ${ }^{1}$, Hervé Chabanne ${ }^{1,2}$, Alain Patey ${ }^{1,2}$ \\ 1 Morpho \\ surname.name@morpho.com \\ 2 Télécom ParisTech \\ surname.name@telecom-paristech.fr \\ Identity and Security Alliance (The Morpho and Télécom ParisTech Research Center)
}

\begin{abstract}
At ISC 2012, Bender et al. introduced the notion of domainspecific pseudonymous signatures for ID documents. With this primitive, a user can sign with domain-specific pseudonyms, that cannot be linked across domains but that are linkable in a given domain. However, their security model assumes non-collusion of malicious users, which is a strong assumption. We therefore propose improvements to their construction. Our main contribution is a new pseudonymous signature scheme based on group signatures that is collusion-resistant.
\end{abstract}

\section{Introduction}

Several security mechanisms [1] are sequentially used when a machine readable travel document (MRTD) connects, via a reader, to a service provider:

- Using Password Authenticated Connection Establishment (PACE), the MRTD and the reader establish a secure channel, once the MRTD user has entered his password;

- Using Extended Acces Control (EAC), the MRTD and the service provider authenticate to each other and establish another secure channel;

- Optionally, using Restricted Identification (RI), the MRTD derives a pseudonym for the service such that the service provider can link the sessions when this particular user accessed the service, but such that it is impossible for two providers to link interactions of one user in their respective domains.

The original Restricted Identification of [1] is close to a Diffie-Hellman key exchange, with static keys, as can be seen in Figure 2. The authenticity of the pseudonym sent by the card is not guaranteed. Furthermore, the domain-specific pseudonym of a user has limited applications, which motivates the work of Bender et al. [3], who suggest to use this pseudonym for digital signatures.

\footnotetext{
* This work has been partially funded by the European FP7 FIDELITY project (SEC2011-284862). The opinions expressed in this document only represent the authors' view. They reflect neither the view of the European Commission nor the view of their employer.

** This paper is an extended version of the eponymous article published at NSS 2013 [6].
} 


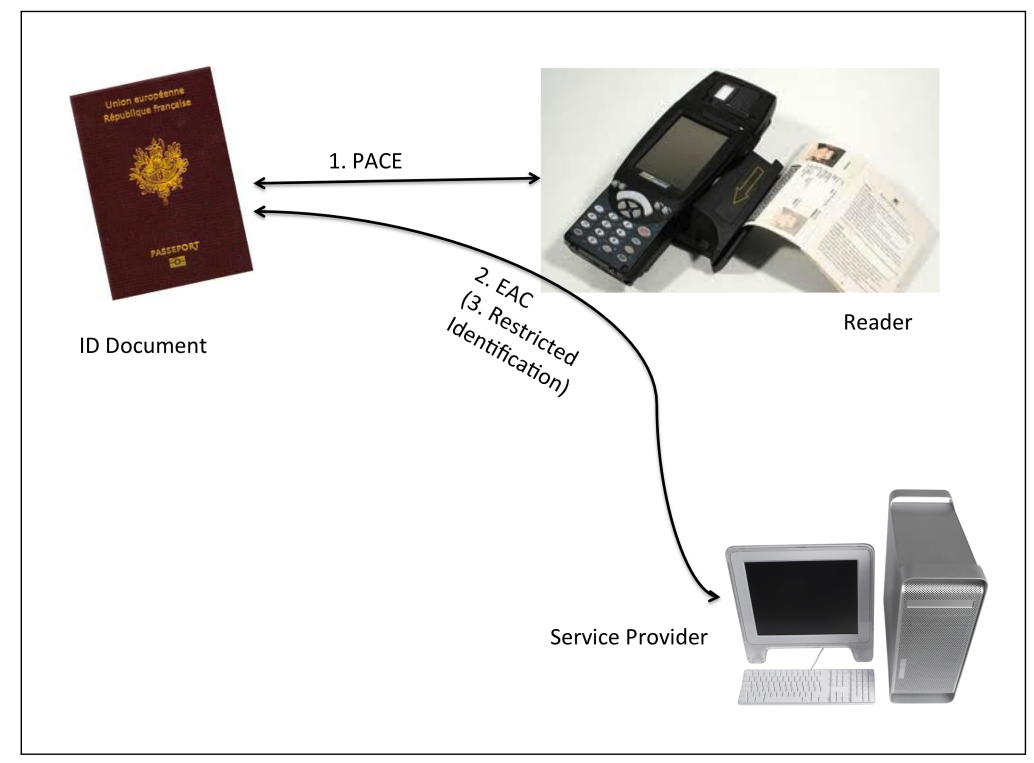

Fig. 1. Advanced Security Mechanisms for MRTDs

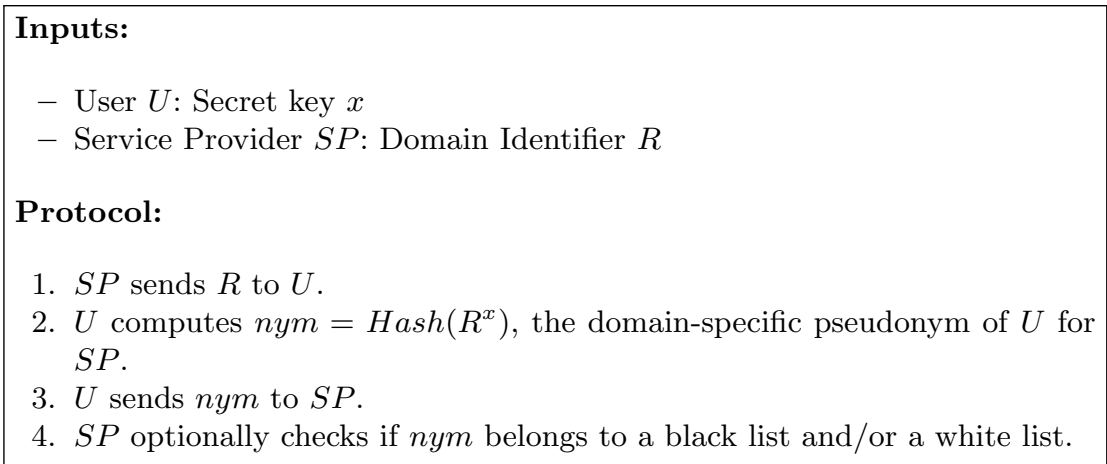

\section{Protocol:}

1. $S P$ sends $R$ to $U$.

2. $U$ computes $n y m=\operatorname{Hash}\left(R^{x}\right)$, the domain-specific pseudonym of $U$ for $S P$.

3. $U$ sends nym to $S P$.

4. $S P$ optionally checks if nym belongs to a black list and/or a white list.

Fig. 2. The Original Restricted Identification Protocol

To augment security guarantees and provide the possibility of using the domain-specific pseudonyms as signature keys, Bender et al. [3] introduced the notion of domain-specific pseudonymous signatures. This can be seen as a relaxation of the notion of group signatures [9,2]. Group signatures enable users to anonymously sign on behalf of a group. Anonymity guarantees are very strong: two signatures of the same user can only be linked by the group manager, who also issues keys. However, pseudonyms produced by the RI protocol for a given user and a given service should enable linkability, since pseudonyms serve as identifiers for the users. 
Domain-specific pseudonymous signatures, as defined in [3], satisfy 3 properties: unforgeability, cross-domain anonymity and seclusiveness. The second property states that pseudonymous signatures cannot be linked across domains while seclusiveness is a relaxation of the notion of traceability [2] for group signatures.

Bender et al. [3] suggest a modification to the original Restricted Identification protocol [1] using pseudonymous signatures, as summed up in Figure 3. In addition to his domain identifier, the service provider sends a message to be signed. This message is signed by the user using a pseudonymous signature. This pseudonymous signature at the same time guarantees that the user owns a valid unrevoked signature key and that the pseudonym is legitimate, i.e. that the same user key has been used to sign and to derive the pseudonym.

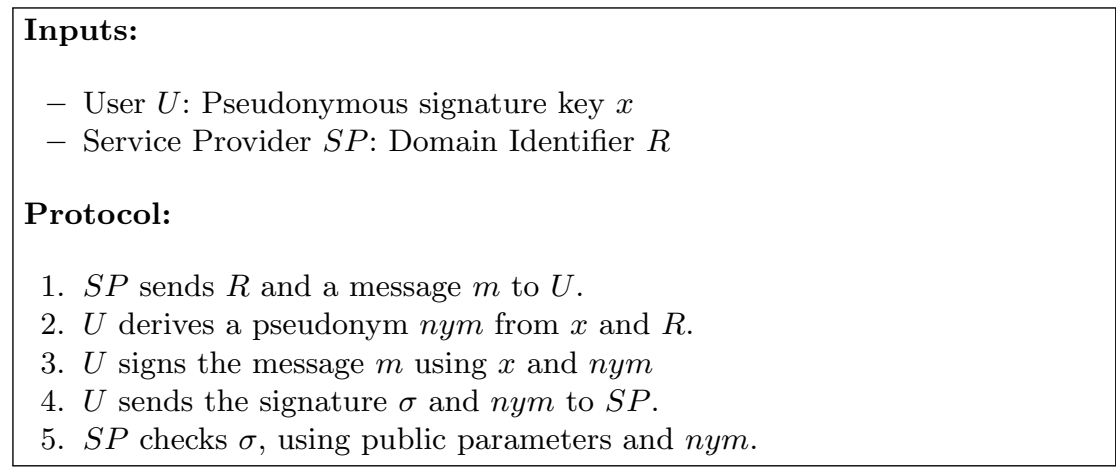

Fig. 3. The Restricted Identification Protocol with Pseudonymous Signatures

The pseudonymous signature scheme of Bender et al. [3] fulfills the security requirements exposed above (unforgeability, cross-domain anonymity and seclusiveness). However, the security model relies on a very strong assumption: they assume that no two malicious users can retrieve their keys and collude. Indeed, the relation between signature keys and issuing key being linear, with two signature keys, one is able to retrieve the issuing key and thus to issue as many new valid keys as they want. To justify their assumption, the authors invoke the fact that these keys are supposed to be stored on smartcards on ID documents, and that smartcards are supposed to be tamper-proof. It is however likely that, on a national scale, two users will be able to retrieve keys stored on their smartcards.

This is the motivation for our work. We propose a new construction of pseudonymous signatures, where we allow the users to access their keys and where collusion of malicious users does not break the security guarantees. To do so, we use (collusion-resistant) group signatures and combine them with Schnorr proofs of knowledge to guarantee validity of pseudonyms. 


\section{Group Signatures}

The generic setting for dynamic group signatures is described in [2]. We here focus on the case of Verifier-Local Revocation (VLR) group signatures [5]. The definitions and properties described in this section may only apply to the VLR case.

\subsection{Setting}

We consider the VLR group signature model [5, 10,8], without Backward Unlinkability. There are three types of entities in the VLR group signature model:

- a Group Manager GM;

- a set of members;

- a set of verifiers.

A VLR Group Signature Scheme with Exculpability consists of the following algorithms:

GSKeyGen $(k)$ : On input a security parameter $k$, this algorithm, run by GM outputs the group public parameters $g p k$ and the issuing key $i k$. It also sets an empty Revocation List $R L$. This list will be later filled with the revocation tokens of the revoked users.

GSJoin $(g p k, i k ; g p k)$ : This algorithm is an interactive protocol between GM and a member $M_{i}$. GM takes as input the public parameters $g p k$ and the issuing key $i k, M_{i}$ only takes $g p k$.

In the end, $M_{i}$ outputs an identity $i d_{i}$, a secret key $s k_{i}$, a credential $c r e_{i}$ and a tracing key $t k_{i}$ (included in $c r e_{i}$ ). GM gets $i d_{i}$ and $t k_{i}$ and outputs also a revocation token $r t_{i}$ for $M_{i}$.

GSRevoke $\left(g p k, r t_{i}, R L\right)$ : GM runs this algorithm to prevent a member $M_{i}$ from making valid signatures. It outputs an updated revocation list $R L$, where $r t_{i}$ has been added.

$\operatorname{GSSign}\left(g p k, s k_{i}\right.$, cre $\left._{i}, m\right)$ : This algorithm, run by a member $M_{i}$, takes as input a message $m, M_{i}$ 's keys $s k_{i}$ and $c r e_{i}$ and a message $m$ to sign. It outputs a signature $\sigma$.

GSVerify $(g p k, R L, m, \sigma)$ : This algorithm, run by a verifier takes as input a message $m$, its signature $\sigma$, the Revocation List $R L$ and the public parameters $g p k$. It checks if the message has been signed by an unrevoked group member, without revealing the signer's identity. The possible outputs are valid and invalid.

GSOpen $\left(g p k, m, \sigma,\left\{t k_{i}\right\}_{i}\right)$ : This algorithm is run by GM. It takes a signature $\sigma$ on a message $m$ as input, together with all tracing keys of the group. It reveals the tracing key $t k_{i}$ and the identity $i d_{i}$ of the signer. If it fails to recover the identity of the signer, it returns $\perp$. 


\section{$2.2 \quad$ Security Requirements}

We require our scheme to satisfy Correctness, Selfless-Anonymity, Traceability and Exculpability. Let us first define Correctness.

Correctness The scheme is correct if every signature created by an unrevoked member is verified as valid.

The other properties are game-based. These games are summed up below.

Selfless-Anonymity Selfless-Anonymity guarantees that no one but the (unrevoked) signer and the GM can gain information about the signer of a given signature.

Consider the following Selfless-Anonymity game played by an adversary $\mathcal{A}$ :

Setup: The challenger $\mathcal{C}$ runs GSKeyGen $(k)$ and obtains $g p k$ and $i k . \mathrm{He}$ sends $g p k$ to $\mathcal{A}$.

Queries: $\mathcal{A}$ can make Join, Sign, Corruption, Revocation and Open queries to the challenger $\mathcal{C}$.

Challenge: Adversary $\mathcal{A}$ outputs a message $m^{*}$ and two members $M_{i_{0}}$ and $M_{i_{1}}$, who are neither corrupted, nor revoked. $\mathcal{C}$ chooses $b \in_{R}\{0,1\}$ and sends $\sigma^{*}=\operatorname{GSSign}\left(g p k, s k_{i_{b}}, \operatorname{cre}_{i_{b}}, m^{*}\right)$ to $\mathcal{A}$.

Restricted Queries: Adversary $\mathcal{A}$ can make the same queries as in the Queries phase, as long as he does not require the revocation or the corruption of $M_{i_{0}}$ or $M_{i_{1}}$, nor the opening of $\sigma^{*}$.

Output: Adversary $\mathcal{A}$ outputs a guess $b^{\prime} \in\{0,1\}$ on $b$.

The scheme is said to satisfy the Selfless-Anonymity property if the probability $\left|\operatorname{Pr}\left[b=b^{\prime}\right]-1 / 2\right|$ is negligible for any probabilistic polynomial-time adversary $\mathcal{A}$.

Traceability The scheme is traceable if an adversary $\mathcal{A}$ is unable to forge a valid signature that cannot be opened properly. Consider the following Traceability game played by $\mathcal{A}$ :

Setup: The challenger $\mathcal{C}$ runs $\operatorname{KeyGen}(k)$ and obtains $g p k$ and $i k$. He sends $g p k$ to $\mathcal{A}$. He also sets an empty revocation list $R L$.

Queries: Adversary $\mathcal{A}$ can execute Join, Corrupt, Sign and Open queries.

Output: Adversary $\mathcal{A}$ outputs a message $m^{*}$ and a signature $\sigma^{*}$.

Adversary $\mathcal{A}$ wins the game if:

1. GSVerify $\left(g p k, R L, m^{*}, \sigma^{*}\right)=$ valid (implying that $\sigma^{*}$ 's opening traces to a member outside the coalition);

2. Adversary $\mathcal{A}$ did not obtain $\sigma^{*}$ by making a Sign query on $m^{*}$.

The scheme satisfies Traceability if no polynomial probabilistic adversary is able to win the above game with a non-negligible probability.

Exculpability The aim of the Exculpability property is to offer protection against the Group Manager. In the Exculpability game, roles are inverted: the adversary is the GM and, consequently, knows the group's secret key and all the players' credentials. The goal of the adversary is to forge a valid signature that will be attributed to an honest (i.e. not corrupted) member by the GSOpen algorithm. This signature must be such that it cannot be denied by the signer. Consider the following Exculpability game played by an adversary $\mathcal{A}$ : 
Setup: Challenger $\mathcal{C}$ runs $G S K e y G e n(k)$ and obtains gpk and $i k . \mathcal{C}$ stores gpk and sends $g p k$ and $i k$ to $\mathcal{A}^{3}$.

Queries: Adversary $\mathcal{A}$ can request Join, Corrupt and Sign queries.

Output: Adversary $\mathcal{A}$ outputs a message $m^{*}$, the Revocation List $R L$, a signature $\sigma^{*}$ and a tracing key pair $\left(i d_{i^{*}}, t k_{i^{*}}\right)$.

Adversary $\mathcal{A}$ wins the game if all the following statements hold:

1. He did not obtain $\sigma^{*}$ from making a query on $m^{*}$;

2. $G S \operatorname{Verify}\left(g p k, R L, m^{*}, \sigma^{*}\right)=$ valid;

3. $\operatorname{GSOpen}\left(g p k, m^{*}, \sigma^{*},\left\{t k_{i}\right\}\right)=\left(i d_{i^{*}}, t k_{i^{*}}\right)$;

4. He did not corrupt $M_{i^{*}}$;

5. It is impossible for $M_{i^{*}}$ to prove the knowledge of a member key $\left(i d_{i}\right.$, $s k_{i}$, cre $\left._{i}\right)$ such that $s k_{i} \neq s k_{i^{*}}$ and such that $s k_{i}$ could have issued $\sigma^{*}$ as a valid signature ${ }^{4}$.

Note that the last requirement is to ensure protection against a dishonest opening from the manager. The scheme satisfies Exculpability if no polynomial probabilistic adversary is able to win the above game with a nonnegligible probability.

\subsection{The CL-BP Scheme}

We here detail the scheme of Chen and Li [10], patched by Bringer and Patey [8] to ensure Traceability, that we denote by CL-BP. This scheme is well-suited for our purpose, since one can efficiently prove that the same key has been used to generate a pseudonym and to sign the message. Furthermore, the CL-BP scheme enjoys efficient revocation checks.

The GSKeyGen algorithm is described in Algorithm 1. The issuing key is $\gamma \in \in_{R} \mathbb{Z}_{p}$, its public counterpart is $w=g_{2}^{\gamma}$.

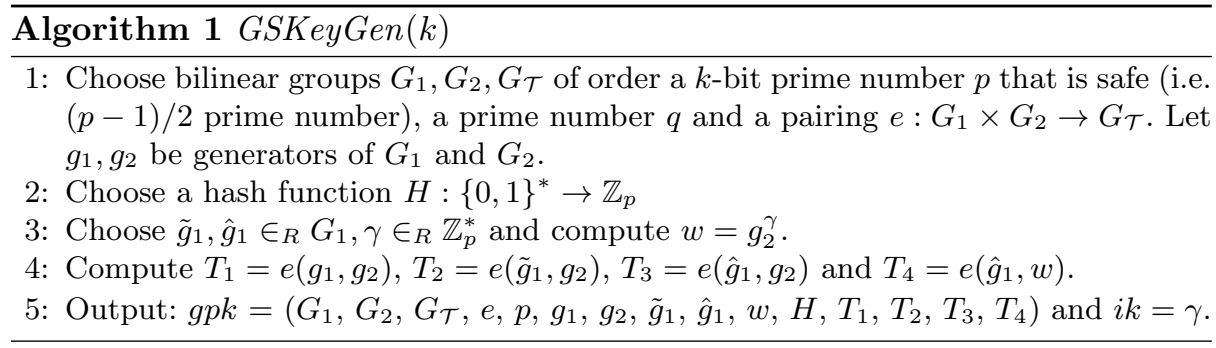

The GSJoin algorithm is explained in Algorithm 2. Each member $M_{i}$ chooses a secret key $s k_{i}=f_{i} \in_{R} \mathbb{Z}_{p}$, not known by GM. $M_{i}$ gives to GM an identity $i d_{i}=\tilde{g}_{1}^{f_{i}}$ and proves the knowledge of $f_{i}$. GM sends him, over a secure channel, a credential cre $_{i}=\left(A_{i}, x_{i}\right)$, that satisfies $e\left(A_{i}, w g_{2}^{x_{i}}\right)=e\left(g_{1} \tilde{g}_{1}^{f_{i}}, g_{2}\right)$. The revocation token for a member $M_{i}$ is $r t_{i}=x_{i}$ and $t k_{i}=x_{i}$ is the tracing key.

\footnotetext{
3 This corresponds to a trusted setup run on behalf of the adversary.

${ }^{4}$ This condition is formalized in [10] by using two functionalities called DProve and DVerify.
} 


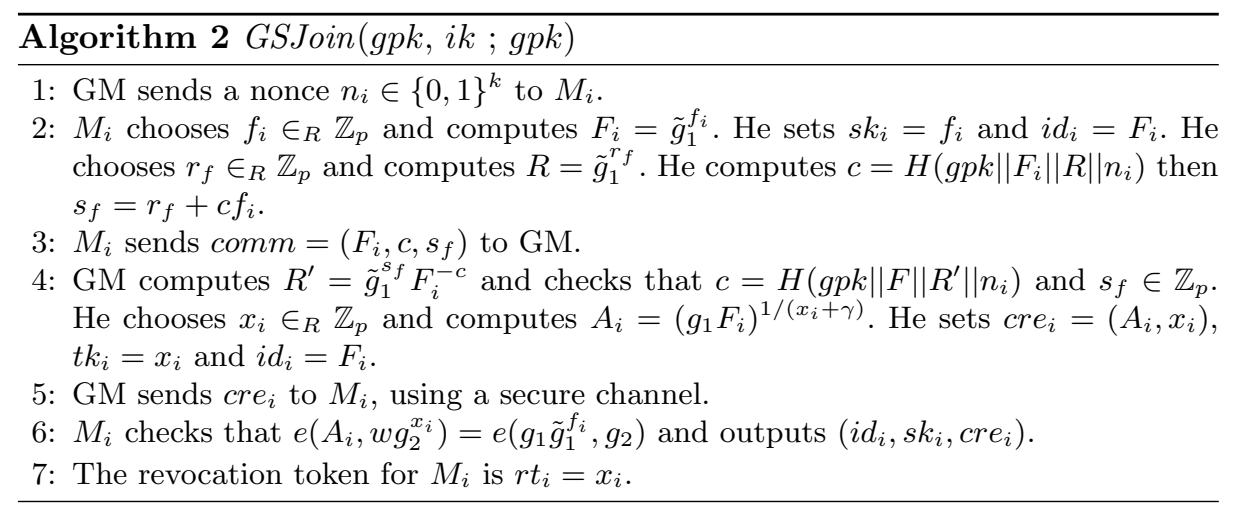

The GSSign algorithm is described in Algorithm 3. When a member $M_{i}$ creates a signature, he first chooses a random $B \in_{R} G_{1}$ and computes $J=B^{f_{i}}$, $K=B^{x_{i}}$. He picks a random $a \in_{R} \mathbb{Z}_{p}$, computes $b=a x_{i}$ and $T=A_{i} \hat{g}_{1}^{a}$. He then does a NIZK PK of $\left(f_{i}, A_{i}, x_{i}\right)$ satisfying $J=B^{f_{i}}, K=B^{x_{i}}$ and $e\left(A_{i}, w g_{2}^{x_{i}}\right)=e\left(g_{1} \tilde{g}_{1}^{f_{i}}, g_{2}\right)$.

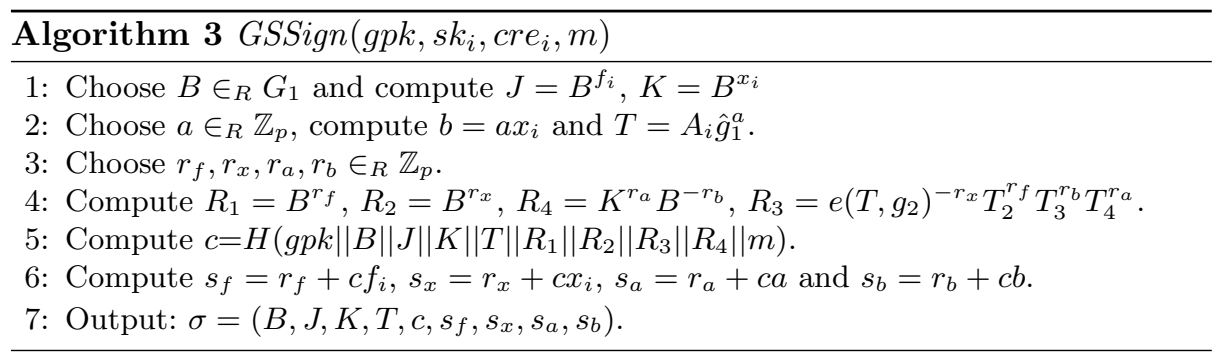

The GSVerify algorithm is described in Algorithm 4. To check a signature, the verifier checks the proof of knowledge. If the check succeeds, he also does a Revocation Check: $\forall r t_{i^{\prime}}=x_{i}^{\prime} \in R L$, he checks that $K \neq B^{r t_{i^{\prime}}}$.

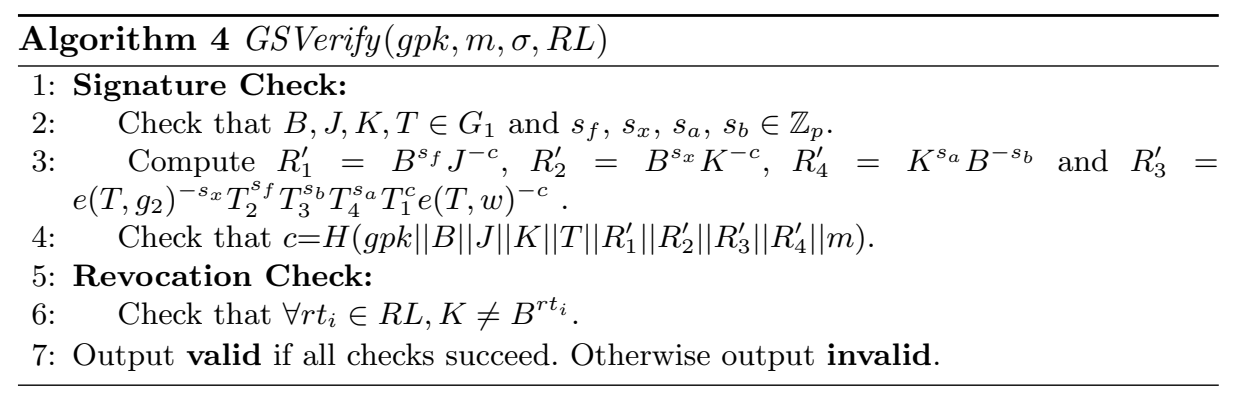


The GSOpen algorithm consists in an application of the Revocation Check part of the GSVerify algorithm, using the revocation tokens of all enrolled users. When there is an equality between $K$ and $B^{r t_{i}}$ (see Algorithm 4, line 6), the corresponding user is output.

The security of the CL-BP scheme is proved in [8]. We sum up the security guarantees in the following theorem, where DDH stands for Decisional DiffieHellman, $q$-SDH for $q$-Strong Diffie Hellman [4] and DLP for Discrete Logarithm Problem.

Theorem 1 (Security of the CL-BP Scheme). The CL-BP scheme achieves

- Correctness;

- Selfless-Anonymity, under the DDH assumption;

- Traceability, under the q-SDH assumption,

- Exculpability, under the DLP assumption.

\section{Pseudonymous Signatures}

We give in this section definitions that follow our stronger security model and indicate where the weaker security model of [3] differs.

\subsection{Setting}

Three entities are involved:

- The Issuing Authority (IA)

- Users $M_{i}$

- Service Providers $S P_{j}$ with specific domains $D_{j}$

The issuing authority issues secret keys to the users and domain parameters to the service providers. The users can generate, from their secret keys and the domain parameters, one pseudonym per domain. Using domain-specific pseudonymous signatures, users can sign messages linked to their pseudonyms. The pseudonym being attached to the signatures, the signatures of one user in one domain are obviously linkable. The construction guarantees that signatures of the same user for different domains are not linkable by (even colluding) service providers. However, the issuing authority can retrieve these links.

There are six algorithms in the scheme:

PSKeyGen $(k)$ The Issuing Authority generates a master secret key $m s k$ and a master public key $g p k$.

The IA and the $S P_{j}$ 's generate domain-specific public keys $d p k_{j}$.

PSJoin $(m s k, g p k)$ The Issuing Authority and the user $M_{i}$ interact to generate a secret key $s k_{i}$ for user $M_{i}$.

$\operatorname{PSSign}\left(g p k, s k_{i}, d p k_{j}, m\right)$ The user $M_{i}$ outputs his pseudonym $n y m_{i j}$ for domain $D_{j}$ and a signature $\sigma$ on $m$ for domain $D_{j}$ 
PSVerify $\left(g p k, n y m_{i j}, d p k_{j}, m, \sigma, R L_{j}\right)$ The Service Provider for domain $D_{j}$ checks the signature $\sigma$ on message $m$ and the link between $\sigma$ and $n y m_{i j} . S P_{j}$ also performs a revocation check, using $R L_{j}$, the list of revoked pseudonyms for domain $D_{j}$

PSDomainRevoke $\left(R L_{j}, n y m_{i j}\right) S P_{j}$ runs this algorithm to prevent a member $M_{i}$ from making valid signatures in $D_{j}$. It outputs an updated revocation list $R L_{j}$, where $n y m_{i j}$ has been added.

PSRevoke $\left(g p k, x_{i}\right)$ IA runs this algorithm to prevent a member $M_{i}$ from making valid signatures in all domains. He sends an information $x_{i}$ about the key of $M_{i}$ that the $S P_{j}$ 's use to revoke $M_{i}$ from their domains. Every service provider $S P_{j}$ outputs an updated $R L_{j}$.

\subsection{Security}

The security properties of pseudonymous signatures are close to the properties of group signatures. We require a pseudonymous signature scheme to satisfy Correctness, Cross-Domain Anonymity, Unforgeability and Seclusiveness.

Correctness The scheme is correct if every signature-pseudonym couple created by an unrevoked member is verified as valid.

Cross-Domain Anonymity Cross-Domain Anonymity guarantees that signatures are anonymous and that linkability is possible within a given domain only, even with colluding service providers.

Consider the following Cross-Domain Anonymity game played by an adversary $\mathcal{A}$ :

Setup: The challenger $\mathcal{C}$ runs KeyGen $(k)$ and obtains gpk and $m s k$. He sends $g p k$ to $\mathcal{A}$.

Queries: $\mathcal{A}$ can make Join, Sign, User Corruption ${ }^{5}$, Service Provider Corruption, Domain-Specific Revocation and Revocation queries to the challenger $\mathcal{C}$.

Challenge: Adversary $\mathcal{A}$ outputs a message $m^{*}$, a domain $D_{j}$ and two members $M_{i_{0}}$ and $M_{i_{1}}$, who are neither corrupted, nor revoked for domain $D_{j}$. Furthermore, no Sign request in the domain $D_{j}$ for these two members must have been called. $\mathcal{C}$ chooses $b \in_{R}\{0,1\}$ and sends $\sigma^{*}=$ $P S \operatorname{Sign}\left(g p k, s k_{i_{b}}, d p k_{j}, m^{*}\right)$ to $\mathcal{A}$.

Restricted Queries: Adversary $\mathcal{A}$ can make the same queries as in the Queries phase, as long as he does not require the revocation or the corruption of $M_{i_{0}}$ or $M_{i_{1}}$, nor signatures of $M_{i_{0}}$ or $M_{i_{1}}$ for domain $D_{j}$.

Output: Adversary $\mathcal{A}$ outputs a guess $b^{\prime} \in\{0,1\}$ on $b$.

The scheme is said to satisfy the Cross-Domain Anonymity property if the probability $\left|\operatorname{Pr}\left[b=b^{\prime}\right]-1 / 2\right|$ is negligible for any probabilistic polynomialtime adversary $\mathcal{A}$.

Seclusiveness The scheme achieves Seclusiveness if an adversary $\mathcal{A}$ is unable to forge a valid signature that cannot be opened properly. Consider the following Seclusiveness game played by $\mathcal{A}$ :

\footnotetext{
${ }^{5}$ In the security definition of the scheme of [3], only one corruption is authorized
} 
Setup: The challenger $\mathcal{C}$ runs $\operatorname{KeyGen}(k)$ and obtains gpk and $i k$. He sends $g p k$ to $\mathcal{A}$. He also sets empty revocation list $R L_{j}$.

Queries: Adversary $\mathcal{A}$ can execute Join, Corrupt ${ }^{6}$, Sign and Open queries.

Output: Adversary $\mathcal{A}$ outputs a message $m^{*}$ and a signature $\sigma^{*}$.

Adversary $\mathcal{A}$ wins the game if:

1. PSVerify $\left(g p k, n y m_{i j}, d p k_{j}, R L_{j}, m^{*}, \sigma^{*}\right)=$ valid (implying that $\sigma^{*}$ 's opening traces to a member outside the coalition);

2. Adversary $\mathcal{A}$ did not obtain $\sigma^{*}$ by making a Sign query on $m^{*}$.

The scheme satisfies Traceability if no polynomial probabilistic adversary is able to win the above game with a non-negligible probability.

Unforgeability The aim of the Unforgeability property is to prevent anyone, including the Group Manager, from making signatures on behalf of a given user. Notice that, contrary to the security definitions of [3], we do not require the group manager to delete information about the users' keys after the PSJoin algorithm.

In the Unforgeability game, roles are inverted: the adversary is the IA and, consequently, knows the group's secret key and all the players' and providers' credentials. The goal of the adversary is to forge a valid signature that will be attributed to an honest (i.e. not corrupted) member. This signature must be such that it cannot be denied by the signer. Consider the following Unforgeability game played by an adversary $\mathcal{A}$ :

Setup: Challenger $\mathcal{C}$ runs $\operatorname{Key} G e n(k)$ and obtains $g p k$ and $i k . \mathcal{C}$ stores $g p k$ and sends $g p k$ and $i k$ to $\mathcal{A}$.

Queries: Adversary $\mathcal{A}$ can request Join, Corrupt and Sign queries.

Output: Adversary $\mathcal{A}$ outputs a message $m^{*}$, a domain $D_{j}$, the Revocation List $R L_{j}$, a signature $\sigma^{*}$, a pseudonym $n y m_{i j}$ and a member $M_{i^{*}}$.

Adversary $\mathcal{A}$ wins the game if all the following statements hold:

1. He did not obtain $\sigma^{*}$ from making a query on $m^{*}$;

2. PSVerify $\left(g p k, n y m_{i j}, d p k_{j}, R L_{j}, m^{*}, \sigma^{*}\right)=$ valid;

3. The signature opens to $M_{i^{*}}$, and $M_{i^{*}}$ cannot deny it.

4. He did not corrupt $M_{i^{*}}$;

The scheme satisfies Unforgeability if no polynomial probabilistic adversary is able to win the above game with a non-negligible probability.

\subsection{Overview of the scheme of Bender et al.}

We sum up the components of the pseudonymous signature scheme of [3] and the reasons why it does not resist to collusions.

Let $G=\langle g\rangle$ be a cyclic group of prime order $q$. The secret key of the IA is made of two randomly chosen integers $x, z \in_{R} \mathbb{Z}_{q}$. The public parameters of the system are $g, g^{x}$ and $g^{z}$.

The key of a user $M_{i}$ is a couple $\left(x_{1 i}, x_{2 i}\right)$, chosen by the IA, such that $x_{1 i}=x-z \cdot x_{2 i}$.

\footnotetext{
${ }^{6}$ In [3], only one Corrupt query is allowed.
} 
The domain parameters for every domain $D_{j}$ are chosen by the IA, by picking a random $r_{j} \in R \mathbb{Z}_{q}^{*}$ and setting $d p k_{j}=g^{r_{j}} . S P_{j}$ learns $d p k_{j}$, but not $r_{j}$.

The pseudonym of a user $M_{i}$ for domain $D_{j}$ is $d p k_{j}^{x_{1 i}}$.

To sign, user $M_{i}$ uses two intertwined proofs of knowledge, a Schnorr proof of knowledge [13] that he knows the discrete logarithm of the pseudonym and an Okamoto proof [12] that he owns a valid $\left(x_{1}, x_{2}\right)$ key pair. The proofs are noninteractive and rely on the random oracle. The message to be signed is included in the entries of the hash function used to generate the challenge.

Limitations First, it is noticeable, that when one finds two $\left(x_{1}, x_{2}\right)$ couples, one easily retrieves the secret keys $x$ and $z$ of the IA and can thus generate as many valid key couples as one wants. The authors of [3] justify that, since these keys are supposed to be stored on smartcards, assumed to be tamper-proof, security will be guaranteed. We estimate that this assumption is optimistic, especially for the sensitive application of ID documents.

Second, the IA generates all the keys and, thus, is able to sign on behalf of the users. It is specified in [3] that these keys are supposed to be deleted by the IA, once delivered to the users, which is impossible to verify. This might raise some security issues, we also solve this problem in our proposal thanks to the Exculpability property of group signatures.

\section{Our Proposal for a Collusion-Resistant Pseudonymous Signature Scheme}

\subsection{The Proposal}

One of the main properties of group signatures is that they prevent from linking signatures. However, pseudonymous signatures are supposed to enable linkability of signatures if they are accompanied by the same pseudonym, but unlinkability otherwise. By combining the construction of [3] with the CL-BP group signature, we build pseudonymous signatures that are resistant to collusions. This construction requires more computation from the user to sign messages. It requires elliptic curve cryptography but the smartcard does not need to be able to compute pairings.

The PSKeyGen algorithm, described in Algorithm 5 is mostly an execution of the GSKeyGen algorithm of the CL-BP scheme by the Issuing Authority. IA also has to pick randomly chosen domain parameters $d p k_{j}=g_{1}^{r_{j}}$ that he sends to the service providers. It is essential, in order to preserve unlinkability, that the service providers do not learn $r_{j}$.

The PSJoin algorithm, described in Algorithm $6^{7}$ consists in an execution of the GSJoin algorithm.

\footnotetext{
${ }^{7}$ Optionally, during the execution of this algorithm, $M_{i}$ also gets $e\left(A_{i}, g_{2}\right)$ to avoid pairing computations by the smartcard
} 

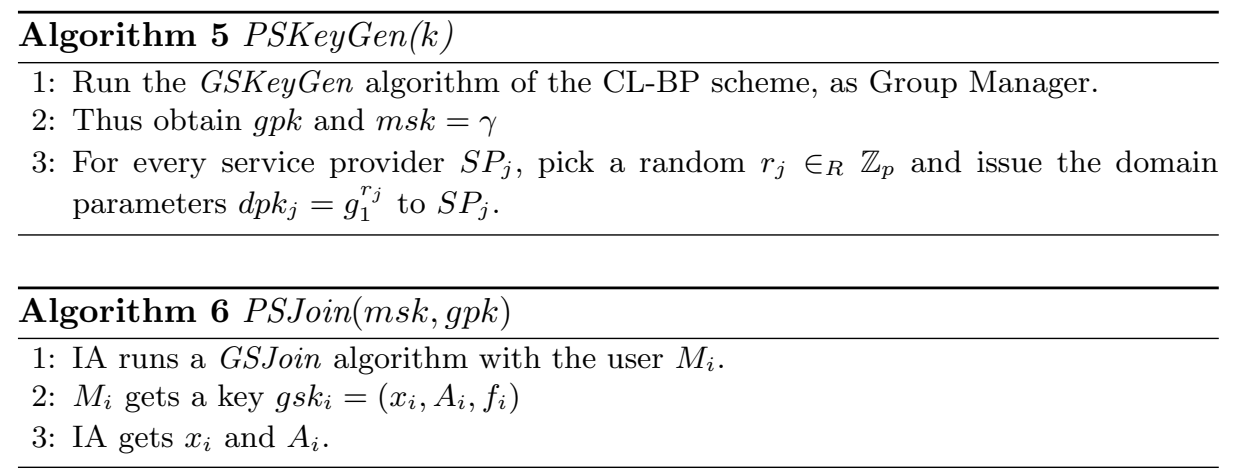

The PSSign algorithm, described in Algorithm 7 consists in a CL-BP signature, a domain-specific pseudonym derivation, and a proof of knowledge that the same key has been used in both operations.

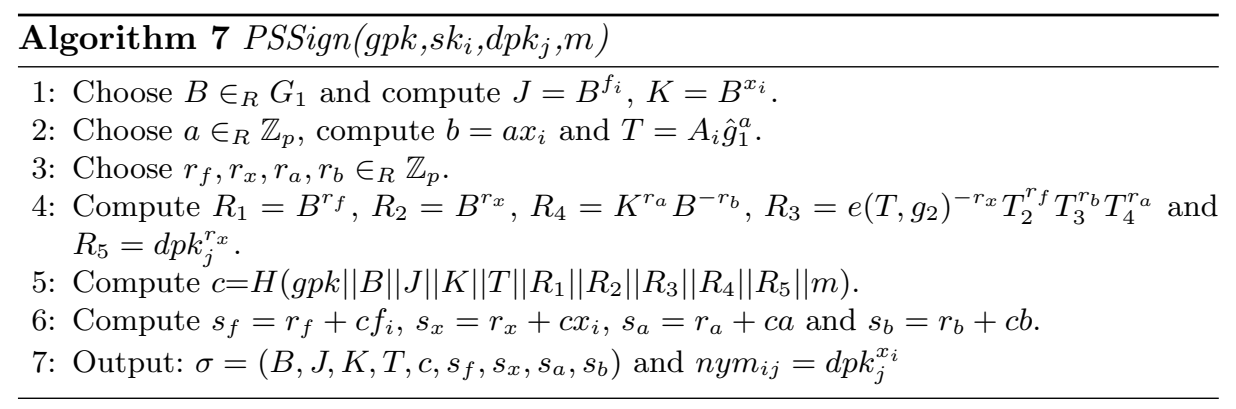

The PSVerify algorithm, described in Algorithm 8 is a verification of the proof of knowledge, followed by a revocation check, where the verifier checks if the pseudonym is on the revocation list or not. Notice that the verifier only performs a list membership test and not a linear number of arithmetic operations, as in the Revocation Check of the GSVerify algorithm.

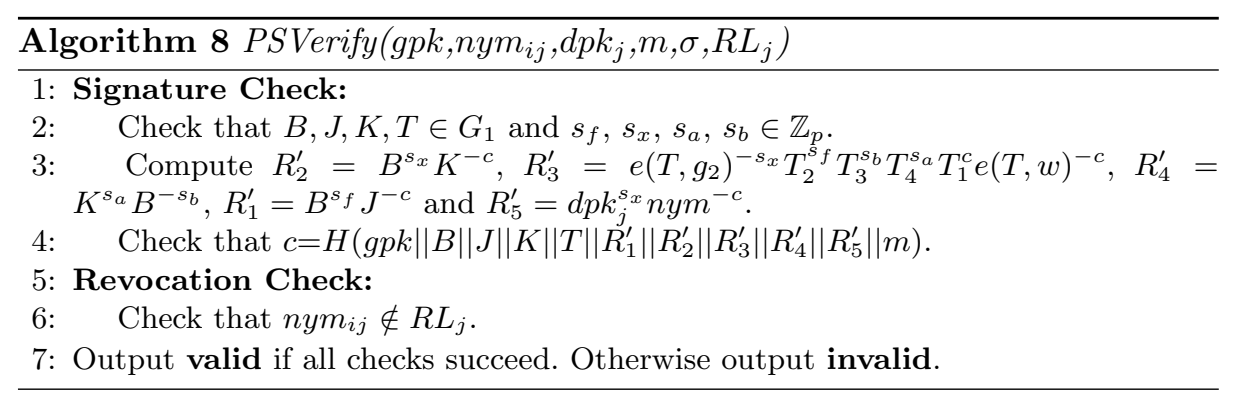


The revocation algorithms, PSDomainRevoke, described in Algorithm 9, and PSRevoke, described in Algorithm 10, consist in adding the pseudonym of the revoked user in the revocation list $R L_{j}$ of the domain $R L_{j}$.
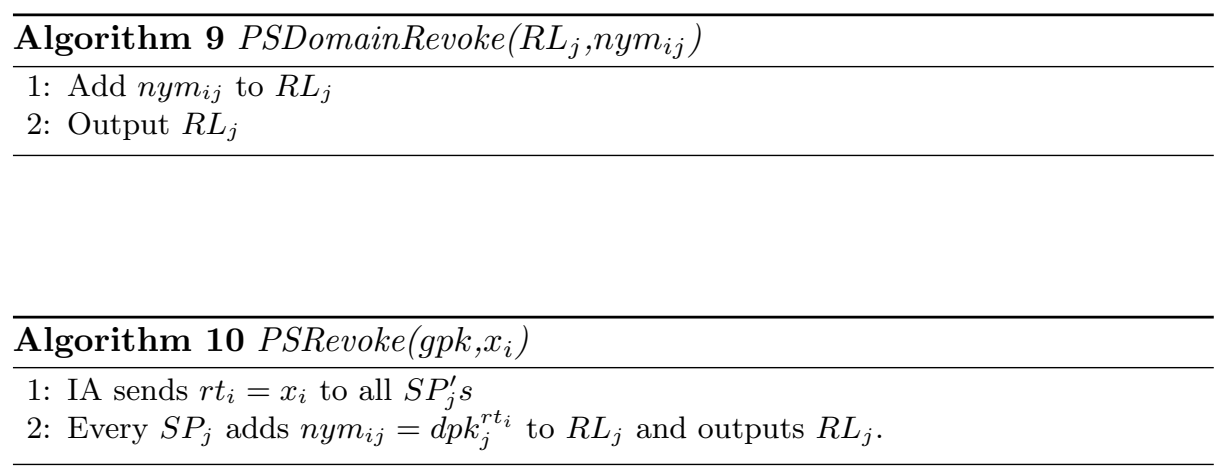

\subsection{Security}

Our pseudonymous signature scheme achieves the security properties described in Section 3.2, under the same security conditions as the CL-BP scheme. It can be proven in a similar way as in $[10,8]$, i.e. by showing how a successful adversary against our security games can be used to break the security assumptions. Security is guaranteed is in the random oracle model.

Theorem 2 (Correctness). The pseudonymous signature scheme described in Section 4.1 achieves Correctness.

Theorem 3 (Cross-Domain Anonymity). Under the Decisional Diffie-Hellman assumption, the pseudonymous signature scheme described in Section 4.1 achieves Cross-Domain Anonymity.

Theorem 4 (Seclusiveness). Under the q-Strong Diffie-Hellman assumption [4], the pseudonymous signature scheme described in Section 4.1 achieves Seclusiveness.

Theorem 5 (Unforgeability). Under the Discrete Logarithm assumption, the pseudonymous signature scheme described in Section 4.1 achieves Unforgeability.

Implementation In [8], parameters for the CL-BP scheme are suggested and a computation time analysis is performed. The computation required by our PSSign algorithm is the cost of the GSSign algorithm of the CL-BP scheme and an exponentiations necessary to compute $R_{5}$ and $n y m_{i j}$. As noticed in [8], all the computation of GSSign but the hash function can be computed offline, before 
the knowledge of the message to be signed. This offline work consists in 6 multiexponentiations in $G_{1}$ and 1 multi-exponentiation in $G_{\mathcal{T}}$. The remaining online work for our pseudonymous signature is then 1 hash function computation and 2 exponentiations in $G_{1}$. With the parameters of [8], this online computation requires less than $100 \mathrm{~ms}$ on a personal computer.

The corresponding computation for PSVerify is 5 multi-exponentiations in $G_{1}, 1$ multi-exponentiation in $G_{\mathcal{T}}$ and 1 pairing. Contrary to the one of GSVerify, the cost of the Revocation Check of PSVerify is negligible. With the parameters of [8], the computation of PSVerify requires around $500 \mathrm{~ms}$ on a personal computer.

\section{Conclusion}

We succeed in building a pseudonymous signature scheme that resists to collusions, which improves on the previous proposal of Bender et al. [3]. This property is an asset for a secure large-scale deployment of this mechanism on electronic ID documents. The price to pay is the use of elliptic-curve cryptography and pairings. However, it can be deployed such that no pairings are computed on the users' smartcards.

Our proposal relying on VLR group signatures, it can be extended to enjoy additional features that have already been proposed for group signatures such as biometric key derivation [7] or Backward Unlinkability [11, 8]. The latter property enables to have a time period-specific revocation process.

\section{References}

1. Advanced security mechanisms for machine readable travel documents. part 2 extended access control version 2 (EACv2), password authenticated connection establishment (PACE), and restricted identification (RI). Tech. Rep. TR-03110-2, BSI (March 2012), version 2.10

2. Bellare, M., Shi, H., Zhang, C.: Foundations of group signatures: The case of dynamic groups. IACR Cryptology ePrint Archive 2004, 77 (2004)

3. Bender, J., Dagdelen, Ö., Fischlin, M., Kügler, D.: Domain-specific pseudonymous signatures for the german identity card. In: Gollmann, D., Freiling, F.C. (eds.) ISC. Lecture Notes in Computer Science, vol. 7483, pp. 104-119. Springer (2012)

4. Boneh, D., Boyen, X.: Short signatures without random oracles. In: Cachin, C., Camenisch, J. (eds.) EUROCRYPT. Lecture Notes in Computer Science, vol. 3027, pp. 56-73. Springer (2004)

5. Boneh, D., Shacham, H.: Group signatures with verifier-local revocation. In: Atluri, V., Pfitzmann, B., McDaniel, P.D. (eds.) ACM Conference on Computer and Communications Security. pp. 168-177. ACM (2004)

6. Bringer, J., Chabanne, H., Patey, A.: Collusion-resistant domain-specific pseudonymous signatures. In: International Conference on Network and System Security (NSS) (2013)

7. Bringer, J., Chabanne, H., Pointcheval, D., Zimmer, S.: An application of the Boneh and Shacham group signature scheme to biometric authentication. In: Matsuura, K., Fujisaki, E. (eds.) IWSEC. Lecture Notes in Computer Science, vol. 5312, pp. 219-230. Springer (2008) 
8. Bringer, J., Patey, A.: VLR group signatures - how to achieve both backward unlinkability and efficient revocation checks. In: SECRYPT. pp. 215-220 (2012)

9. Chaum, D., van Heyst, E.: Group signatures. In: Davies, D.W. (ed.) EUROCRYPT. Lecture Notes in Computer Science, vol. 547, pp. 257-265. Springer (1991)

10. Chen, L., Li, J.: VLR group signatures with indisputable exculpability and efficient revocation. In: Elmagarmid, A.K., Agrawal, D. (eds.) SocialCom/PASSAT. pp. 727-734. IEEE Computer Society (2010)

11. Nakanishi, T., Funabiki, N.: A short verifier-local revocation group signature scheme with backward unlinkability. In: Yoshiura, H., Sakurai, K., Rannenberg, K., Murayama, Y., ichi Kawamura, S. (eds.) IWSEC. Lecture Notes in Computer Science, vol. 4266, pp. 17-32. Springer (2006)

12. Okamoto, T.: Provably secure and practical identification schemes and corresponding signature schemes. In: Brickell, E.F. (ed.) CRYPTO. Lecture Notes in Computer Science, vol. 740, pp. 31-53. Springer (1992)

13. Schnorr, C.P.: Efficient signature generation by smart cards. J. Cryptology 4(3), 161-174 (1991) 